\title{
Micromechanical magnetometer using an all-silicon nonlinear torsional resonator
}

\author{
D. Antonio, ${ }^{\text {a),b) }}$ M. I. Dolz, ${ }^{\text {a) }}$ and H. Pastoriza ${ }^{\text {a) }}$ \\ Centro Atómico Bariloche, 8400 S. C. de Bariloche, Argentina
}

(Received 30 July 2009; accepted 10 September 2009; published online 1 October 2009)

\begin{abstract}
In this work, a micromagnetometer employing a nonlinear torsional resonator with a high quality factor $Q$ is presented experimentally. Oscillatory rotation of a conducting plate in the sensed magnetic field $H$ induces eddy currents that dissipate energy. Due to the nonlinear response of the oscillator, the resulting mechanical damping originates frequency shifts in the resonance curve that depend on $H$. Nonlinearity results from the electrostatic detection, which introduces high order electrical spring constants. The device is fabricated with a standard silicon process and does not incorporate ferromagnetic materials. An analytical nonlinear model that correctly describes the device is also introduced. (C) 2009 American Institute of Physics. [doi:10.1063/1.3242003]
\end{abstract}

The use of micromachined sensors for measuring magnetic fields offers the benefits of reduced size, low cost, high sensitivity, direct integration with electronics, and low power. However, these devices generally require attached or deposited magnetic materials to interact with the magnetic field. These materials are not standard in microelectromechanical systems fabrication processes and they add complexity to the design. ${ }^{1-3}$ Moreover, with those devices, the quantitative interpretation of results is not straightforward. For example, using resonators with deposited ferromagnetic films to measure magnetization requires knowing the saturation magnetization and anisotropy constants of the film, and fitting the frequency shift curve with an appropriate function. ${ }^{4}$

Greywall ${ }^{5}$ theoretically presented a novel magnetometer that incorporates a nonlinear mechanical resonator. This sensor does not require magnetic materials and is appropriate for fabrication with standard micromachining processes. When the resonator is driven into resonance and a magnetic field is applied, the induced currents originate damping in the mechanical system. If the resonator is nonlinear, this damping produces significant variations in the peak frequency that can be measured with good sensitivity due to its high $Q$.

We propose in this work an implementation of this idea with a torsional micromechanical resonator. The nonlinearity is originated by the electrostatic actuation and detection, and the mechanical energy is dissipated by eddy-type currents induced by the rotation of the device in the magnetic field to be measured.

We present experimentally the response of the sensor to changes in the magnetic field, thus demonstrating the applicability of the operating principles proposed by Greywall. Additionally we introduce an analytical model based in our previous work ${ }^{6}$ that correctly describes the device in the linear and nonlinear regimes. The model takes in account the electrostatic excitation and detection, and incorporates the operation dependence with the applied magnetic field.

The polycrystalline silicon (PolySi) mechanical microresonator, ${ }^{6}$ based on an original design by Bolle et al. ${ }^{7}$

\footnotetext{
${ }^{a}$ Member of: Consejo Nacional de Investigaciones Científicas y Técnicas (CONICET), Argentina.

${ }^{b}$ Electronic mail: dario.antonio@cab.cnea.gov.ar.
}

was fabricated in the MEMSCAP (Ref. 8) foundry using its multiuser PolyMUMPs process. The structure consists of a $106 \times 44 \mu \mathrm{m}^{2}$ suspended central plate that is anchored to the substrate by means of two serpentine springs. The plate's thickness is $1.5 \mu \mathrm{m}$ and the gap between the plate and the underlying electrodes is $2.75 \mu \mathrm{m}$. One of these electrodes is used to electrostatically actuate the oscillator while the other is used to detect its motion by measuring variations of capacitance between the plate and the electrode by means of a cryogenic transimpedance preamplifier. ${ }^{9}$

We have shown in a previous work ${ }^{6}$ that when this torsional microresonator is driven by a weak periodic forcing it obeys the Duffing equation:

$$
I \frac{d^{2} \theta}{d t^{2}}+\frac{I \omega_{0}}{Q} \frac{d \theta}{d t}+k_{1} \theta+k_{3} \theta^{3}=\tau_{o} \cos (\omega t),
$$

where $\theta$ is the angle of oscillation, $I$ is the moment of inertia, $k_{1}$ and $k_{3}$ are the linear and nonlinear elastic constants, $\omega_{0}$ $=\sqrt{k_{1} / I}$ is the natural frequency, and $\tau_{o} \cos (\omega t)$ is the excitation torque. For this device, the linear elastic constant $k_{1}$ is mainly determined by the geometry of the springs and the mechanical properties of the PolySi, and only slightly modified by the electrostatic detection voltage bias. In contrast, the nonlinear elastic constant $k_{3}$ is exclusively originated by this voltage. All the parameters in Eq. (1) can be evaluated from the dimensions, elastic properties, and voltage levels involved, except the quality factor, which needs to be measured or estimated.

In the case of weakly nonlinear forced oscillations, the solution to Eq. (1) can be approximated by a sinusoidal response of the form $\theta \approx \theta_{m} \cos (\omega t+\varphi)$, where $\theta_{m}$ and $\varphi$ can be found by solving a cubic algebraic system of equations. ${ }^{10}$

In addition, for an oscillator described by Eq. (1) it can be shown ${ }^{11}$ that

$$
\begin{aligned}
& \omega_{p}=\omega_{0}\left(1+\frac{3}{8} \frac{k_{3} \theta_{p}^{2}}{I \omega_{0}^{2}}\right), \\
& \theta_{p}=\theta_{0} Q,
\end{aligned}
$$

where $\theta_{p}$ is the maximum oscillation amplitude of the resonance curve, which occurs at the peak frequency $\omega_{p}$, and $\theta_{0}=\tau_{0} /\left(I \omega_{0}^{2}\right)$ is the oscillation amplitude for $\omega \ll \omega_{0}$. 


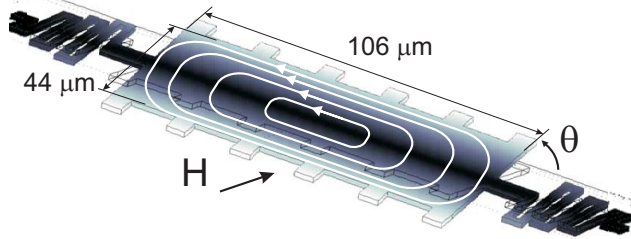

FIG. 1. (Color online) Eddy currents induced in the conducting plate as it oscillates in the magnetic field $H$.

Therefore, changes in the energy dissipated by the microoscillator produce a variation of its quality factor and its oscillation amplitude. This amplitude variation does not affect $\omega_{p}$ in the linear operating range, but if the microoscillator is working in the nonlinear range it produces a variation of $\omega_{p}$ given by Eq. (2).

If a magnetic field $H$ is applied parallel to the plate and perpendicular to the rotation axis of the resonator the oscillating perpendicular component of the field induces eddy currents in the plate as sketched in Fig. 1. These currents originate Ohmic losses in the PolySi and damp the resonator's motion. For the PolySi the electromagnetic skin depth is much larger than the dimensions of the plate and we can consider that the magnetic field is constant. Given this condition we can calculate the energy $E_{h}$ dissipated by the eddy currents in one cycle of the oscillator. Approximating the rectangular plate by a square plate of side $a$ with the same thickness $b=1.5 \mu \mathrm{m}$ and surface $a^{2}=(106 \times 44 \mu \mathrm{m})^{2}$ $=(68.3 \mu \mathrm{m})^{2}$, and following the procedure described in Ref. 12 , we find

$$
E_{h}=\frac{a^{4} b H^{2} \pi \theta_{m}^{2} \omega}{32 \rho}
$$

where $\rho=(30 \pm 15) \times 10^{-6} \Omega \mathrm{m}$ is the electrical resistivity of the plate.

The total quality factor $Q$ has a contribution $Q_{h}$ from these losses induced by the magnetic field, and a contribution $Q_{i}$ from the intrinsic losses of the oscillator,

$$
\begin{aligned}
& Q^{-1}=Q_{h}^{-1}+Q_{i}^{-1}, \\
& Q_{h}=2 \pi \frac{E_{\max }}{E_{h}}=\frac{32 \rho k_{1}}{a^{4} b H^{2} \omega},
\end{aligned}
$$

where $E_{\max }$ is the maximum energy stored per cycle.

Therefore Eqs. (3), (5), and (6) relate the peak amplitude in the resonance to the applied magnetic field. Combined with Eq. (2) they finally give us the theoretical dependence of the peak frequency $\omega_{p}$ with $H$.

Experimentally, we have used two different configurations to investigate the nonlinear microoscillator as a magnetometer. The first one is an open loop configuration where the excitation frequency is swept in order to record the amplitude and phase response of the microoscillator near the resonance. In the second configuration, the microoscillator is set to work in a certain point of the resonance curve. In our case this is done by adjusting the driving frequency using a proportional-integral-derivative controller (PID) computer program in order to maintain the phase of the detected signal in a fixed value, or set point.

With the open loop configuration, we obtained the resonance curves shown in Fig. 2, with a driving voltage $V_{\text {ac }}$

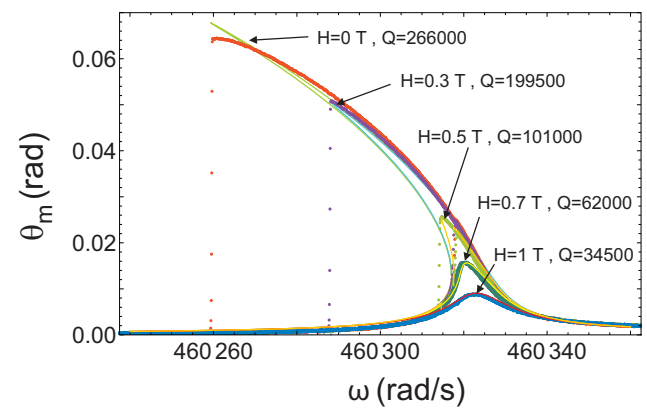

FIG. 2. (Color online) Resonance curves for $V_{\mathrm{ac}}=160 \mathrm{mV}$ and different values of $H$. The theoretical curves are showed with solid lines. The agreement between the model and the experiment is excellent for oscillation amplitudes lower than 0.03 rad.

$=160 \mathrm{mV}$ and for different values of the applied magnetic field. The measurements were made at a temperature $T$ $=10 \mathrm{~K}$ since the quality factor of this resonator is maximum at temperatures near liquid helium $(Q \approx 250000)$. In the same figure, we show the solutions of Eq. (1) (solid lines). The theoretical solutions show the unstable region of the curves. For $\theta_{p}<0.05 \mathrm{rad}$, the amplitude curves calculated with the model are in excellent agreement with the measured ones but for larger values of the oscillation amplitude this agreement deteriorates. This is due to the fact that the model is valid only for weakly nonlinear oscillations driven by a weak periodic forcing. ${ }^{6}$

The closed loop operation is the normal operating mode of the magnetometer. The original idea in Ref. 5 was to keep the system oscillating in the peak frequency, which depends on the peak amplitude and thus follows the variations of the applied magnetic field. In our measurements we track the frequency $\nu_{\varphi=\varphi_{0}}$ for which the phase difference between drive and response has a specified value $\varphi_{0}$. If $\varphi_{0}=\pi / 2$ these two conditions are equivalent. The measured variation of $\nu_{\varphi=\varphi_{0}}$ as a function of the applied magnetic field is shown in Fig. 3 for three different excitation voltages. The values of $\varphi_{0}$ were chosen as close as possible to $\pi / 2$ but differing from it by a value large enough to prevent the jump to the other branch of the hysteresis loop. With this feedback configuration we were not able to achieve operation in the open loop's unstable region. A refinement of the design should

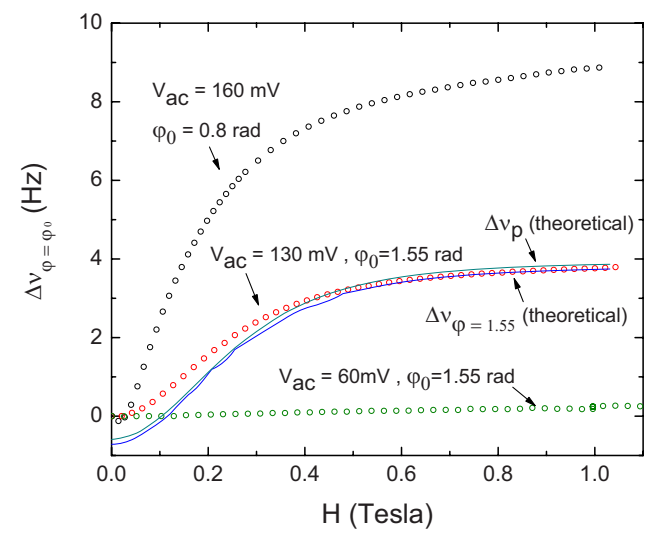

FIG. 3. (Color online) Measured variations of the constant phase frequency, vs applied magnetic field, for different excitation voltages. For $V_{\text {ac }}$ $=60 \mathrm{mV}$ the system is in the linear range and there is almost no frequency variation with magnetic field. The theoretical curves corresponding to $\Delta \nu_{p}$ and $\Delta \nu_{\varphi=1.55}$ are shown for $V_{\mathrm{ac}}=130 \mathrm{mV}$. 
make this possible since it has been demonstrated that stable operation is attainable at any point of the resonance curve if the proper feedback is used. ${ }^{13}$ However, working at the peak frequency is not strictly necessary for the correct operation of the device.

It can be seen in Fig. 3 how the variation of the frequency $\Delta \nu_{\varphi=\varphi_{0}}$ for a given change in the applied magnetic field increases with increasing excitation voltages. For $V_{\text {ac }}$ $=60 \mathrm{mV}$ the response of the oscillator is almost linear and there is no variation of $\nu_{\varphi=\varphi_{0}}\left(\right.$ or $\left.\omega_{p}\right)$ with $H$.

In the same figure, we included two additional curves derived from the theoretical model and corresponding to an excitation voltage of $130 \mathrm{mV}$. The first one, labeled $\Delta \nu_{p}$, was obtained by combining Eqs. (3), (5), and (6) with Eq. (2) in order to find the theoretical relation between the peak frequency $\nu_{p}$ and the applied field. The second curve was obtained by solving Eq. (1) with the method described in ${ }^{6}$ in order to find $\Delta \nu_{\varphi=1.55}$. The similarity between the two curves proves that following the frequency corresponding to the peak amplitude is approximately equivalent to following the frequency at which the phase has a value $\varphi \approx \pi / 2$. However the second theoretical curve strictly represents our experiment and, accordingly, it gives the best fit to the measured data. The agreement deteriorates for $H<0.4 \mathrm{~T}$ because the oscillation amplitudes are larger than required for applying the model.

In conclusion, we have presented a micromachined magnetometer which does not require magnetic materials and is fabricated with a standard PolySi fabrication process. The nonlinear operation of the device and its dependence with an applied magnetic field is correctly described by an analytical model. The largest frequency variation in Fig. 3 was obtained with the maximum excitation voltage $V_{\mathrm{ac}}=160 \mathrm{mV}$, for which $\Delta \nu_{\varphi=\varphi_{0}} / \Delta H \approx 37 \mathrm{~Hz} / \mathrm{T}$ at $H=0.1 \mathrm{~T}$. This sensitivity does not change much down to $H=0.03 \mathrm{~T}$ and the field of maximum sensitivity can still be reduced by increasing the plate's conductivity. ${ }^{5}$ Considering that the minimum detect- able change in frequency with this setup is $0.01 \mathrm{~Hz}$ we can detect field changes of $0.3 \mathrm{mT}$ with $H=0.1 \mathrm{~T}$. At this stage our effort was not focused on high sensitivity but on demonstrating the feasibility of this type of sensor. Nevertheless, ameliorations to the device could be easily introduced. For instance, gold coating of the plate is available in this fabrication process and would increase $E_{h} 300$ times for any given $H$. Room temperature operation would reduce $Q$ $(\approx 3000)$ but larger excitation voltages could also be used, which would increase the nonlinearity and therefore $\Delta \nu_{\varphi=\varphi_{0}} / \Delta H$. Finally, the feedback electronics can be improved to lower the minimum detectable frequency variation.

We acknowledge fruitful discussions with Daniel López and support from ANPCyT under Grant No. PAE2004 \# 22592.

${ }^{1}$ J. Moreland, J. Phys. D: Appl. Phys. 36, R39 (2003).

${ }^{2}$ H. H. Yang, N. V. Myung, J. Yee, D. Y. Park, B. Y. Yoo, M. Schwartz, K. Nobe, and J. W. Judy, Sens. Actuators, A 97, 88 (2002).

${ }^{3}$ R. P. Cowburn, A. M. Moulin, and M. E. Welland, Appl. Phys. Lett. 71, 2202 (1997)

${ }^{4}$ M. I. Dolz, W. Bast, D. Antonio, H. Pastoriza, J. Curiale, R. D. Sánchez, and A. G. Leyva, J. Appl. Phys. 103, 083909 (2008).

${ }^{5}$ D. S. Greywall, Meas. Sci. Technol. 16, 2473 (2005).

${ }^{6}$ D. Antonio and H. Pastoriza, J. Microelectromech. Syst. (to be published). ${ }^{7}$ C. A. Bolle, V. Aksyuk, F. Pardo, P. L. Gammel, E. Zeldov, E. Bucher, R. Boie, D. J. Bishop, and D. R. Nelson, Nature (London) 399, 43 (1999).

${ }^{8}$ MEMSCAP Inc., 4021 Stirrup Creek Drive, Durham, NC 27703, USA, http://www.memscap.com

${ }^{9}$ D. Antonio, H. Pastoriza, P. Julián, and P. Mandolesi, Rev. Sci. Instrum. 79, 084703 (2008).

${ }^{10}$ J. Guckenheimer and P. Holmes, Nonlinear Oscillations, Dynamical Systems, and Bifurcations of Vector Fields, Applied Mathematical Sciences, Vol. 42, 2nd ed. (Springer, New York, 1986).

${ }^{11}$ B. Yurke, D. S. Greywall, A. N. Pargellis, and P. A. Busch, Phys. Rev. A 51, 4211 (1995).

${ }^{12}$ A. F. Kip, Fundamentals of Electricity and Magnetism, Fundamentals of Modern Physics, 1st ed. (Mc Graw-Hill, New York, 1962).

${ }^{13}$ D. S. Greywall, B. Yurke, P. A. Busch, A. N. Pargellis, and R. L. Willett, Phys. Rev. Lett. 72, 2992 (1994). 